\title{
The $\alpha$-dependence of transition frequencies for ions Si II, Cr II, Fe II, Ni II, and Zn II
}

\author{
V. A. Dzuba ${ }^{1}$, V. V. Flambaum ${ }^{1}$, M. G. Kozlov 母 $^{\text {and M. Marchenko }}{ }^{1}$ \\ 1 University of the New South Wales, Sydney, Australia and \\ 2 Petersburg Nuclear Physics Institute, Gatchina, 188300, Russia
}

(Dated: March 22, 2022)

\begin{abstract}
We performed accurate calculation of $\alpha$-dependence $\left(\alpha=e^{2} / h c\right)$ of the transition frequencies for ions, which are used in a search for the variation of the fine structure constant $\alpha$ in space-time. We use Dirac-Hartree-Fock method as a zero approximation and then the many-body perturbation theory and configuration interaction methods to improve the results. An important problem of level pseudocrossing (as functions of $\alpha$ ) is considered. Near the crossing point the derivative of frequencies over $\alpha$ varies strongly (including change of the sign). This makes it very sensitive to the position of the crossing point. We proposed a semiempirical solution of the problem which allows to obtain accurate results.
\end{abstract}

PACS numbers: 31.30.Jv, 06.20.Jr, 95.30.Dr

\section{INTRODUCTION}

Recently there was an intensive discussion of the possible space-time variation of the fine structure constant $\alpha=e^{2} / h c$ at the cosmological scale. The first evidence for such variation has been reported in [1, 2, 3, 4, , 5, 6] from the analysis of the astrophysical data. These results are to be compared with the number of experimental upper bounds on this variation obtained from other astrophysical observations (see, e.g. 7, 8, 99|) and from the precision laboratory measurements [10, 11, 12]. Recently a number of new laboratory tests have been proposed (see, e.g. [13]). The analysis of the microwave background radiation can also give some restrictions on time variation of $\alpha$ as suggested in [14, 15, 16]. Implementations of the space-time variation of the fine structure constant to the theory of the fundamental interactions are discussed e.g. in Refs. 17, 18, 19, 20, 21, 22, 23] (see also disscussion and references in $[3]$ ).

The most straitforward way to look for the variation of $\alpha$ is to measure the ratio of some fine structure interval to an optical transition frequency, such as $\omega\left(n p_{1 / 2} \rightarrow\right.$ $\left.n p_{3 / 2}\right)$ and $\omega\left(n^{\prime} s_{1 / 2} \rightarrow n p_{3 / 2}\right)$ [37]. This ratio can be roughly estimated as $0.2 \alpha^{2} Z^{2}$, where $Z$ is the nuclear charge 24]. Therefore, any difference in this ratio for a laboratory experiment and a measurement for some distant astrophysical object can be easily converted into the space-time variation of $\alpha$. However, as it was pointed out in [25], one can gain about an order of magnitude in the sensitivity to the $\alpha$-variation by comparing optical transitions for different atoms. In this case the frequency of each transition can be expanded in a series in $\alpha^{2}$ :

$$
\begin{aligned}
\omega_{i} & =\omega_{i}^{(0)}+\omega_{i}^{(2)} \alpha^{2}+\ldots \\
& =\omega_{i, \text { lab }}+q_{i} x+\ldots, \quad x \equiv\left(\alpha / \alpha_{0}\right)^{2}-1,
\end{aligned}
$$

where $\alpha_{0}$ stands for the laboratory value of the fine struc-

*Electronic address: mgk@MF1309.spb.edu ture constant. Note, that Eq. (1a) corresponds to the expansion at $\alpha=0$, while Eq. (1b) - to the expansion at $\alpha=\alpha_{0}$. In both cases parameters $\omega_{i}^{(2)}$ and $q_{i}$ appear due to relativistic corrections.

For a fine structure transition the first coefficient on the right hand side of (1a) turns to zero, while for the optical transitions it does not. Thus, for the case of a fine structure and an optical transition one can write:

$$
\frac{\omega_{\mathrm{fs}}}{\omega_{\mathrm{op}}}=\frac{\omega_{\mathrm{fs}}^{(2)}}{\omega_{\mathrm{op}}^{(0)}} \alpha^{2}+O\left(\alpha^{4}\right),
$$

while for two optical transitions $i$ and $k$ the ratio is:

$$
\frac{\omega_{i}}{\omega_{k}}=\frac{\omega_{i}^{(0)}}{\omega_{k}^{(0)}}+\left(\frac{\omega_{i}^{(2)}-\omega_{k}^{(2)}}{\omega_{k}^{(0)}}\right) \alpha^{2}+O\left(\alpha^{4}\right) .
$$

Quite often the coefficients $\omega_{i}^{(2)}$ for optical transitions are about an order of magnitude larger than corresponding coefficients for the fine structure transitions $\omega_{\mathrm{fs}}^{(2)}$ (this is because the relativistic correction to a ground state electron energy is substantially larger than the spin-orbit splitting in an excited state [25, 26]). Therefore, the ratio (3) is, in general, more sensitive to the variation of $\alpha$ than the ratio (2). It is also important that the signs of coefficients $\omega_{i}^{(2)}$ in (3) can vary. For example, for $s-p$ transitions the relativistic corrections are positive while for $d-p$ transitions they are negative. This allows to suppress possible systematic errors which "do not know" about the signs and magnitude of the relativistic corrections [25]. On the other hand, for many cases of interest, the underlying atomic theory is much more complicated for Eq. (3). In particular, the most difficult case corresponds to transitions to highly excited states of a multielectron atom, where the spectrum is very dense. And this happens to be a typical situation for astrophysical spectra, in particular, for large cosmological red shifts. Corresponding atomic calculations have to account very accurately for the electronic correlations, which may affect such spectra quite dramatically. 
TABLE I: Final results for parameters $q$ from Eq. (1) for Si II, Cr II, Fe II, Ni II, and Zn II. Estimated errors are in brackets.

\begin{tabular}{cccr}
\hline \hline Ion & Transition & $\omega_{0}\left(\mathrm{~cm}^{-1}\right)$ & \multicolumn{1}{c}{$q\left(\mathrm{~cm}^{-1}\right)$} \\
\hline Si II ${ }^{2} P_{1 / 2}^{o}$ & $\rightarrow{ }^{2} D_{3 / 2}$ & 55309.3365 & $520(30)$ \\
& $\rightarrow{ }^{2} S_{1 / 2}$ & 65500.4492 & $50(30)$ \\
\hline Cr II ${ }^{6} S_{5 / 2}$ & $\rightarrow{ }^{6} P_{3 / 2}^{o}$ & 48398.868 & $-1360(150)$ \\
& $\rightarrow{ }^{6} P_{5 / 2}^{o}$ & 48491.053 & $-1280(150)$ \\
& $\rightarrow{ }^{6} P_{7 / 2}^{o}$ & 48632.055 & $-1110(150)$ \\
\hline Fe II ${ }^{6} D_{9 / 2}$ & $\rightarrow{ }^{6} D_{9 / 2}^{o}$ & 38458.9871 & $1330(150)$ \\
& $\rightarrow{ }^{6} D_{7 / 2}^{o}$ & 38660.0494 & $1490(150)$ \\
& $\rightarrow{ }^{6} F_{11 / 2}^{o}$ & 41968.0642 & $1460(150)$ \\
& $\rightarrow{ }^{6} F_{9 / 2}^{o}$ & 42114.8329 & $1590(150)$ \\
& $\rightarrow{ }^{6} P_{7 / 2}^{o}$ & 42658.2404 & $1210(150)$ \\
& $\rightarrow{ }^{4} F_{7 / 2}^{o}$ & 62065.528 & $1100(300)$ \\
& $\rightarrow{ }^{6} P_{7 / 2}^{o}$ & 62171.625 & $-1300(300)$ \\
\hline Ni II ${ }^{2} D_{5 / 2}$ & $\rightarrow{ }^{2} F_{7 / 2}^{o}$ & 57080.373 & $-700(250)$ \\
& $\rightarrow{ }^{2} D_{5 / 2}^{o}$ & 57420.013 & $-1400(250)$ \\
& $\rightarrow{ }^{2} F_{5 / 2}^{o}$ & 58493.071 & $-20(250)$ \\
\hline Zn II ${ }^{2} S_{1 / 2}$ & $\rightarrow{ }^{2} P_{1 / 2}^{o}$ & 48481.077 & $1584(25)$ \\
& $\rightarrow{ }^{2} P_{3 / 2}^{o}$ & 49355.002 & $2490(25)$ \\
\hline \hline
\end{tabular}

The first calculations of the coefficients $q$ from Eq. (1) for the transitions suitable for astronomical and laboratory measurements were done in Refs. 25, 26, 27, 28]. Here we present a new and more accurate calculations of the coefficients $q$ for the transitions, which are currently used in the analysis of the astrophysical data. A full list of these transitions was given in [3]. We have not recalculated here the lightest and the most simple atoms $\mathrm{Mg}$ and $\mathrm{Al}$, for which the previous calculation [25] should be sufficiently accurate and focused on more complicated ions Si II, Cr II, Fe II, Ni II, and Zn II. Our final results for them are given in Table \ Note, that here we use the single parameter $q$ instead of two parameters $q_{1}$ and $q_{2}$ used in the earlier works and $q \equiv \partial \omega /\left.\partial x\right|_{x=0}=q_{1}+2 q_{2}$. Details of the calculations and discussion of the accuracy will be given in Sec. III] Before that we briefly address few theoretical points in Sec. III.

\section{THEORY}

In order to find parameters $q=\partial \omega /\left.\partial x\right|_{x=0}$ in Eq. (1) we perform atomic calculations for three values of $x$ : $x_{-}=-1 / 8, x_{0}=0$, and $x_{+}=1 / 8$. That allows us to determine $q: q=4\left(\omega\left(x_{+}\right)-\omega\left(x_{-}\right)\right)$and also estimate the second derivative $\partial^{2} \omega /\left.\partial x^{2}\right|_{x=0}$. The large value of the latter signals that interaction between levels is strong (level pseudocrossing), and there is a risk of large errors. For these cases further analysis was done as described below.

a. Relativistic calculations of multi-electron ions. In order to accurately account for the dominant relativistic effects we use the Dirac-Hartree-Fock approximation as a starting point for all calculations of atomic spectra. Though most of the calculations were done for the
Coulomb potential, we have also estimated Breit corrections by including the magnetic part of the Breit interaction in the self-consistent field 29].

The ions we are dealing with in this paper have from one to nine electrons in the open shells. For one valence electron in Zn II the Dirac-Fock $V^{N-1}$ approximation already gives rather good results. On the next step the core-valence correlations can be accounted for by means of the many-body perturbation theory (MBPT). Already the second order MBPT correction allows to reproduce the spectrum with the accuracy, better than $1 \%$, which is more than sufficient for our current purposes.

Other ions of interest to us have at least three valence electrons. Here the dominant correlation correction to transition frequencies corresponds to the valence-valence correlations. This type of correlations can be accounted for with configuration interaction (CI) method. If necessary, the core-valence correlations can be included within combined CI+MBPT technique [30]. The latter usually provides an accuracy of the order of $1 \%$ or better for the lower part of the spectra of atoms and ions with two or three valence electrons [30, 31, 32]. However, the accuracy of $a b$ initio methods decreases with the number of valence electrons and with excitation energy. Indeed, for a large number of valence electrons and/or sufficiently high excitation energy the spectrum becomes dense and the levels with the same exact quantum numbers strongly interact with each other. The part of the spectrum of $\mathrm{Fe}$ II above $55000 \mathrm{~cm}^{-1}$ and, to a somewhat lesser extent, the spectrum of Ni II represent this situation. Therefore, for these ions we developed a semiempirical fitting procedure, which is described below.

In order to have additional control of the accuracy of our CI we performed calculations for most of the ions with two different computer packages. One package was used earlier in Refs. 30, 32, 33 and another one was used in Refs. [3, 25, 26, 27, 28, 31]. The former package allows to construct flexible basis sets and optimize configuration space, while the latter allows for a larger CI space as it works with the block of the Hamiltonian matrix, which corresponds to a particular total angular momentum of atom $J$. When there were no significant difference between two calculations, we only give results obtained with the first package. Nevertheless, our final results presented in Table $\llbracket$ are based on both calculations.

b. Semiempirical treatment of the strong interaction of levels: pseudo-crossing. In the nonrelativistic limit $\alpha \rightarrow 0$, all multi-electron states are accurately described by the $L S$-coupling scheme: $E_{\alpha \rightarrow 0}=E_{p, n, L, S, J}$, where $p= \pm 1$ is the parity and $n$ numerates levels with the same $p, L, S$, and $J$. For sufficiently small values of $\alpha$ the $L S$-coupling holds and the energy has the form:

$$
\begin{aligned}
& E_{p, n, L, S, J}=E_{p, n, L, S}^{(0)}+\left(\frac{\alpha}{\alpha_{0}}\right)^{2}\left(C_{p, n, L, S}\right. \\
& \left.+\frac{1}{2} A_{p, n, L, S}[J(J+1)-L(L+1)-S(S+1)]\right),
\end{aligned}
$$


where the first term in the parentheses gives the slope for the centre of the multiplet and the second term gives the fine structure. With growing $\alpha$ the multiplets start to overlap and when the levels with the same $p$ and $J$ come close, the pseudo-crossing takes place.

Near the pseudo-crossing the slope of the energy curves changes dramatically. If such crossing takes place at $x \approx$ 0 , where $x$ is defined by Eq. (11), i.e. near the physical value of $\alpha$, it can cause significant uncertainty in the values of parameters $q$.

Let us first analyze the behaviour of the slopes $q(x)$ in the vicinity of the pseudo-crossing in the two-level approximation. Consider two levels $E_{1}$ and $E_{2}$ which cross at $x=x_{c}$ :

$$
\begin{aligned}
& E_{1}=q_{1}\left(x-x_{c}\right), \\
& E_{2}=q_{2}\left(x-x_{c}\right) .
\end{aligned}
$$

If the interaction matrix element between these two levels is $V$, the exact adiabatic levels will be

$$
\begin{aligned}
E_{a, b} & =\frac{1}{2}\left(\left(q_{1}+q_{2}\right)\left(x-x_{c}\right)\right. \\
& \left. \pm \sqrt{\left(q_{1}-q_{2}\right)^{2}\left(x-x_{c}\right)^{2}+4 V^{2}}\right) .
\end{aligned}
$$

It is easy now to calculate the energy derivative in respect to $x$ in terms of the mixing angle $\phi$ between unperturbed states 1 and 2:

$$
\frac{\partial E_{a, b}}{\partial x}=\cos ^{2} \phi q_{1,2}+\sin ^{2} \phi q_{2,1} .
$$

Note, that at the crossing the angle $\phi$ varies from 0 on one side through $\pi / 4$ in the centre to $\pi / 2$ on the other side, which leads to the change of the slope $q_{a}(x)=\partial E_{a} / \partial x$ from $q_{1}$ through $\left(q_{1}+q_{2}\right) / 2$ to $q_{2}$. The narrow crossings with small $V$ are particularly dangerous, as the slopes change very rapidly within the interval $\Delta x \approx V /\left|q_{1}-q_{2}\right|$. Then, even small errors in the position of the crossing point $x_{c}$, or the value of $V$ can cause large errors in $q_{a, b}$. In this model we assume that nondiagonal term $V=$ const. For the real atom $V \propto \alpha^{2}$. However, if the crossing region $\Delta x \ll 1$, we can neglect the dependence of $V$ on $\alpha$.

c. Semiempirical treatment of the strong interaction of levels: multi-level case. Eq. (7) can be easily generalized to a multi-level case as it simply gives the slope of a physical level $a$ as a weighted average of the mixed levels. Thus, if the level $a$ can be expressed as a linear combination of some unperturbed $L S$-states $\psi_{L_{n}, S_{n}}$ :

$$
|a\rangle=\sum_{n} C_{n}\left|\psi_{L_{n}, S_{n}}\right\rangle,
$$

the resultant slope $q_{a}$ is given by:

$$
q_{a}=\sum_{n} C_{n}^{2} q_{n} .
$$

Here again we neglect weak dependence of interaction $V$ on $x$ in comparison to strong dependence of $C_{n}^{2}$ on $x$ near crossing points.

Eq. (9) allows to improve $a b$ initio coefficients $q$ if we can find the expansion coefficients $C_{n}$ in Eq. (8). That can be done, for example, by fitting $g$-factors. The magnetic moment operator $\boldsymbol{\mu}=g_{0}(\boldsymbol{L}+2 \boldsymbol{S})$ is diagonal in $L$ and $S$ and, therefore, does not mix different $L S$-states. Thus, in the $L S$-basis the resultant $g$-factor for the state $a$ has exactly the same form as $q_{a}$ :

$$
g_{a}=\sum_{n} C_{n}^{2} g_{n}
$$

If the experimental $g$-factors are known, one can use Eq. (10) to find weights $C_{n}^{2}$ and, then find the corrected values of the slopes $q_{a}$.

Sometimes, the experimental data on g-factors are incomplete. Than, one can still use a simplified version of Eqs. (9) and (10):

$$
\begin{aligned}
& g_{a}=C^{2} g_{a}^{0}+\left(1-C^{2}\right) \bar{g}, \Rightarrow C^{2}=\frac{g_{a}-\bar{g}}{g_{a}^{0}-\bar{g}}, \\
& q_{a}=C^{2} q_{a}^{0}+\left(1-C^{2}\right) \bar{q} .
\end{aligned}
$$

$C^{2}$ here is the weight of the dominant $L S$-level in the experimental one, and the bar means the averaging over the admixing levels. Of course, there is some arbitrariness in calculation of averages $\bar{g}$ and $\bar{q}$. However, the advantage of Eqs. (11) is that only one experimental $g$-factor is required.

\section{DETAILS OF THE CALCULATION AND RESULTS}

As we mentioned above, we performed calculations of energy levels for three values of the parameter $x: x_{-}=$ $-1 / 8, x_{0}=0$, and $x_{+}=1 / 8$. All three calculations were done at exactly same level of approximation, to minimize the error caused by the incompleteness of the basis sets and configuration sets. From these calculations we found two approximations for $q$ : $q_{-}=8\left(\omega\left(x_{0}\right)-\omega\left(x_{-}\right)\right)$and $q_{+}=8\left(\omega\left(x_{+}\right)-\omega\left(x_{0}\right)\right)$. If there were problems with level identification we performed additional calculation for $x=$ 0.01 , where the $L S$-coupling should be very accurate and identification is straitforward. The noticeable difference between $q_{-}$and $q_{+}$signaled the possibility of the level crossing. In these cases we applied the semiempirical procedure described in Sec.【to find the corrected values for $q$; otherwise, we simply took the average: $q=\left(q_{+}+\right.$ $\left.q_{-}\right) / 2$.

\section{A. Zn II}

Zn II has the ground state configuration $\left[1 s^{2} \ldots 3 d^{10}\right] 4 s$ and we are interested in the $4 s \rightarrow 4 p_{j}$ transitions. As the theory here is much simpler than for other ions, we 
TABLE II: Transition frequencies and parameters $q$ for $\mathrm{Zn}$ II (in $\mathrm{cm}^{-1}$ ). Calculations were done in four different approximations: Dirac-Hartree-Fock-Coulomb (DHFC), DiracHartree-Fock-Coulomb-Breit (DHFCB), Brueckner-Coulomb (BC), and Brueckner-Coulomb-Breit (BCB).

\begin{tabular}{rrrrrr}
\hline \hline Transition & Exper. & DHFC & DHFCB & BC & BCB \\
\hline \multicolumn{5}{c}{ transition frequencies } \\
$4 s_{1 / 2} \rightarrow 4 p_{1 / 2}$ & 48481.077 & 44610.1 & 44608.1 & 48391.2 & 48389.4 \\
$\rightarrow 4 p_{3 / 2}$ & 49355.002 & 45346.9 & 45330.0 & 49263.8 & 49244.6 \\
\hline \multicolumn{5}{c}{ parameters $q=\left(q_{+}+q_{-}\right) / 2$} \\
$4 s_{1 / 2} \rightarrow 4 p_{1 / 2}$ & 1362 & 1359 & 1594 & 1590 \\
$\rightarrow 4 p_{3 / 2}$ & 2129 & 2109 & 2500 & 2479 \\
\hline \hline
\end{tabular}

used Zn II to study the importance of the core-valence correlation correction and Breit correction to the slopes $q$. The former correction was calculated in Brueckner approximation:

$$
\left(H_{\mathrm{DHF}}+\Sigma(E)\right) \Psi=E \Psi,
$$

with the self-energy operator $\Sigma(E)$ calculated in the second order of MBPT (the perturbation here is the difference between the exact and Dirac-Hartree-Fock Hamiltonians, $\left.V=H-H_{\mathrm{DHF}}\right)$. The $H_{\mathrm{DHF}}$ was calculated with the magnetic part of the Breit operator included self-consistently. The retardation part of the Breit operator is known to be significantly smaller [29] and we completely neglected it here.

The results of our calculations of the frequencies $\omega$ and the slopes $q$ for two transitions $4 s \rightarrow 4 p_{j}, j=1 / 2,3 / 2$ are given in Table III One can see, that both BruecknerCoulomb and Brueckner-Coulomb-Breit approximations give very good transition frequencies, accurate to $0.2 \%$, though the latter slightly underestimates the fine splitting. Breit correction to the parameters $q$ does not exceed $1 \%$, while core-valence correlations account for the $17 \%$ correction.

In Table II we did not give separately the values of $q_{ \pm}$. The difference between them is close to $1 \%$. Indeed, in the absence of close interacting levels the dependence of $q$ on $x$ arise from the corrections to the energy of the order of $\alpha^{4} Z^{4}$, which are very small.

\section{B. Si II}

Si II has three valence electrons and the ground state configuration $\left[1 s^{2} \ldots 2 p^{6}\right] 3 s^{2} 3 p$. Excited configurations of interest are $3 s 3 p^{2}$ and $3 s^{2} 4 s$. We made the CI calculation in the Coulomb approximation on the basis set, which included $1 s-8 s, 2 p-8 p, 3 d-8 d$, and $4 f, 5 f$ orbitals, which we denote as the basis set [8spd5f]. Note, that we use virtual orbitals, which are localized within the atom [34], rather than Dirac-Fock ones. This provides fast convergence. CI included all single-double (SD) and partly triple excitations from three valence configurations listed above. The results of these calculations are given in Table
TABLE III: Transition frequencies $\omega$ from the ground state ${ }^{2} P_{1 / 2}^{o}$, fine structure splitting $\Delta_{\mathrm{FS}}$, and parameters $q_{ \pm}$for Si II (in $\mathrm{cm}^{-1}$ ).

\begin{tabular}{lrrrrrr}
\hline \hline & \multicolumn{3}{c}{ Experiment [35] } & \multicolumn{4}{c}{ Theory } \\
& $\omega$ & $\Delta_{\mathrm{FS}}$ & \multicolumn{1}{c}{$\omega$} & $\Delta_{\mathrm{FS}}$ & $q_{-}$ & $q_{+}$ \\
\hline${ }^{2} P_{3 / 2}^{o}$ & 287 & 287 & 293 & 293 & 295 & 291 \\
${ }^{4} P_{1 / 2}$ & 44080 & & 41643 & & 453 & 451 \\
${ }^{4} P_{3 / 2}$ & 44191 & 111 & 41754 & 111 & 565 & 564 \\
${ }^{4} P_{5 / 2}$ & 44364 & 174 & 41935 & 181 & 746 & 744 \\
${ }^{2} D_{3 / 2}$ & 55304 & & 54655 & & 509 & 507 \\
${ }^{2} D_{5 / 2}$ & 55320 & \multirow{2}{*}{16} & 54675 & 20 & 530 & 530 \\
${ }^{2} S_{1 / 2}$ & 65495 & & 65148 & & 40 & 39 \\
\hline \hline
\end{tabular}

Like in $\mathrm{Zn}$, the left and write derivatives $q_{-}$and $q_{+}$ are close to each other, and all levels with equal exact quantum numbers are well separated. The astrophysical data exist for the levels ${ }^{2} S_{1 / 2}$ and ${ }^{2} D_{5 / 2}$. The former corresponds to the $3 p \rightarrow 4 s$ transition and has small slope $q$, while the latter corresponds to the $3 s \rightarrow 3 p$ transition and has much larger positive $q$. That is in agreement with the fact, that relativistic corrections to the energy usually decrease with the principle quantum number $n$ and with the orbital quantum number $l$. Therefore, for the $n s \rightarrow n p$ transition one should expect large and positive $q$, while for $n p \rightarrow(n+1) s$, there should be large cancellation of relativistic corrections to upper and to lower levels, resulting in smaller $q$ (see disscussion in 25, 26] ]). The dominant correction to our results should be from the core-valence correlations. In the recent calculations of $\mathrm{Mg}$, which has the same core as Si II, the core-valence corrections to transition frequencies were found to be about $4 \%[33,36]$. We conservatively estimate corresponding correction to $q$ to be $6 \%$ of the larger $q$, i.e. $30 \mathrm{~cm}^{-1}$.

\section{Cr II}

Cr II has the ground state configuration $\left[1 s^{2} \ldots 3 p^{6}\right] 3 d^{5}$ with five valence electrons. The astrophysical data correspond to the $3 d \rightarrow 4 p$ transition, for which one may expect negative value of $q$. CI calculations here are much more complicated, than for Si II. There is strong relaxation of the $3 d$ shell in the discussed transition, which requires more basic $d$-orbitals. Therefore, we used the [6sp9d6f] basis set. In CI we included only single and double (SD) excitations. Some of the triple, quadruple, and octuple excitations were accounted for by means of the second order perturbation theory. It was found that corresponding corrections to transition frequencies were of the order of few percent, and were even smaller for parameters $q$. In general, these corrections did not improve the agreement with the experiment, so we present only CI results in Table IV

As we mentioned above, there is strong relaxation of the $3 d$-shell in the $3 d \rightarrow 4 p$ transition. We were not able to saturate CI space and completely account for this 
TABLE IV: Transition frequencies $\omega$ from the ground state ${ }^{6} S_{5 / 2}$, fine structure splitting $\Delta_{\mathrm{FS}}$, and parameters $q$ for Cr II (in $\mathrm{cm}^{-1}$ ). CI single-double approximation was used for the Coulomb-Breit interaction.

\begin{tabular}{lcrrrr}
\hline \hline & \multicolumn{3}{c}{ Experiment } & \multicolumn{3}{c}{ Theory } \\
& $\omega$ & $\Delta_{\mathrm{FS}}$ & $\omega$ & $\Delta_{\mathrm{FS}}$ & $q_{+}$ \\
\hline${ }^{6} D_{5 / 2}$ & 12148 & & 13123 & & -2314 \\
${ }^{6} D_{7 / 2}$ & 12304 & 156 & 13289 & 165 & -2153 \\
${ }^{6} F_{1 / 2}^{o}$ & 46824 & & 47163 & & -1798 \\
${ }^{6} F_{3 / 2}^{o}$ & 46906 & 82 & 47244 & 81 & -1715 \\
${ }^{6} F_{5 / 2}^{o}$ & 47041 & 135 & 47378 & 134 & -1579 \\
${ }^{6} F_{7 / 2}^{o}$ & 47228 & 187 & 47565 & 187 & -1387 \\
${ }^{6} F_{9 / 2}^{o}$ & 47465 & 237 & 47803 & 238 & -1148 \\
${ }^{6} F_{11 / 2}^{o}$ & 47752 & 287 & 48091 & 288 & -862 \\
${ }^{6} P_{1 / 2}^{o}$ & 48399 & & 48684 & & -1364 \\
${ }^{6} P_{2 / 2}^{o}$ & 48491 & 92 & 48790 & 106 & -1278 \\
${ }^{6} P_{3 / 2}^{o}$ & 48632 & 141 & 48947 & 157 & -1108 \\
\hline \hline
\end{tabular}

relaxation. Because of that, we estimate the error for $q$ here to be close to $10 \%$.

We have seen before for Zn II and Si II, that in the absence of level-crossing the difference between $q_{+}$and $q_{-}$is smaller than other theoretical uncertainties. In $\mathrm{Cr}$ II there are no close levels which may interact with each other, so in the calculation presented in Table IV we determined only the right derivative $q_{+}$. In calculations with different basis sets we checked that the difference between $q_{+}$and $q_{-}$is much smaller than the given above theoretical error (see Table 【).

\section{Fe II}

Fe II ion has 7 valence electrons in configuration $3 d^{6} 4 s$ and represents the most complicated case. The astrophysical data includes 5 lines in the band $38000 \mathrm{~cm}^{-1}$ $43000 \mathrm{~cm}^{-1}$ and two lines with the frequency close to $62000 \mathrm{~cm}^{-1}$. The first band consists of three close, but separated multiplets with a regular fine structure splittings. The $62000 \mathrm{~cm}^{-1}$ band is completely different as the multiplets here strongly overlap and fine structure intervals are irregular [35]. Characteristic distance between the levels with identical exact quantum numbers is few hundred $\mathrm{cm}^{-1}$, which is comparable to the fine structure splittings. This means that the levels strongly interact and even their identification may be a problem.

In fact, in Moore Tables 35] one of the multiplets of interest, namely $y^{6} P^{o}$, is erroneously assign to the configuration $3 d^{6}\left({ }^{7} S\right) 4 p$. It is an obvious misprint, as there is no term ${ }^{7} S$ for configuration $3 d^{6}$. This term appears, however, in the configuration $3 d^{5}$ and the correct assignment of this multiplet should be $3 d^{5}\left({ }^{7} S\right) 4 s 4 p$. This assignment is in agreement with our calculations and with the experimental $g$-factor of the level with $J=7 / 2$. We checked that all close levels of the configuration $3 d^{6} 4 p$ have significantly smaller $g$-factors.

This reassignment has dramatic consequences in terms

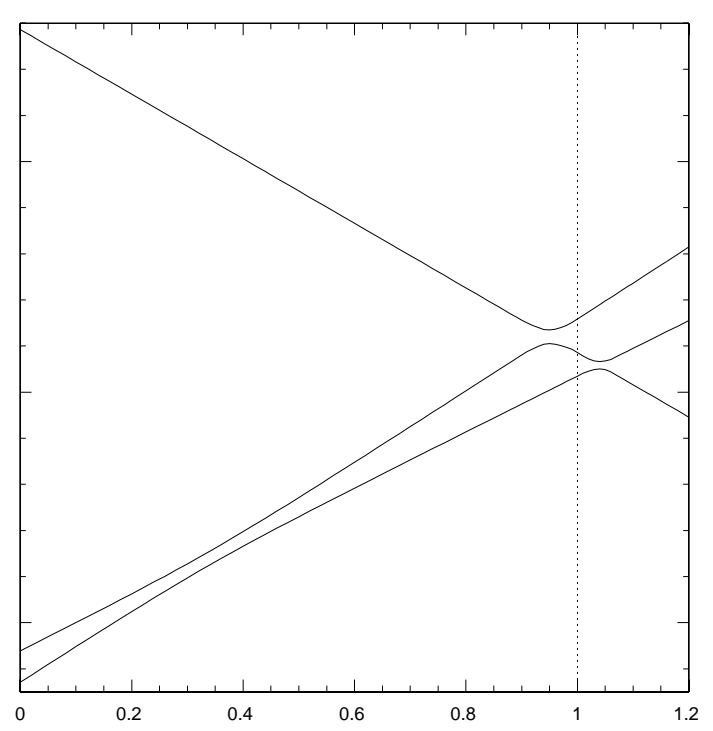

FIG. 1: Examples of typical interaction of levels in the upper band of Fe II. Levels are shown in arbitrary units as function of $\left(\alpha / \alpha_{0}\right)^{2}=x+1$. Levels of configuration $3 d^{6} 4 p$ have similar slopes and strongly interact with each other. That causes wide pseudo-crossings, similar to one shown on the left side of the plot. The level ${ }^{6} P_{7 / 2}^{o}$ of the configuration $3 d^{5} 4 s 4 p$ moves in the opposite direction. A series of sharp pseudo-crossings takes place near the physical value of $\alpha$, marked by a vertical dotted line.

of the corresponding parameter $q$ as configurations $3 d^{6} 4 p$ $\left(4 s-4 p\right.$ transition from the ground state) and $3 d^{5} 4 s 4 p$ ( $3 d-4 p$ transition) move in the opposite directions from the ground state configuration $3 d^{6} 4 s$ when $x$ is changed. It also causes a number of pseudo-crossings to occur right in the vicinity of $x=0$ (see Fig. 11).

CI calculations for Fe II were done on the basis set [6spdf] in the SD approximation (see Table $\mathbf{\nabla}$. Triple excitations were included within second order perturbation theory and corresponding corrections were found to be relatively small. One can see from Table $\nabla$ that for the lower band both frequencies and $g$-factors are reproduced rather accurately.

The first anomaly takes place at $44000 \mathrm{~cm}^{-1}$, where the levels ${ }^{4} D_{7 / 2}^{o}$ and ${ }^{4} F_{7 / 2}^{o}$ appear in the reverse order. Theoretical $g$-factors are also much further from $L S$ values (1.429 and 1.238). That means that theoretical levels are at pseudo-crossing, while experimental levels already passed it. Indeed, calculations for $x=1 / 8$ show that the right order of levels is restored, though the $g$-factors are still too far from $L S$ values.

The second anomaly corresponds to the band above $60000 \mathrm{~cm}^{-1}$. Here the order of calculated levels differs from that of the experimental ones. Note, that for this band only levels of negative parity with $J=7 / 2$ are given in Table $\mathbf{D}$ Thus, all of them can interact with each other. Let us estimate, how this interaction can 
TABLE V: Transition frequencies $\omega$ from the ground state ${ }^{6} D_{9 / 2}, g$-factors, and parameters $q_{ \pm}$for Fe II $\left(\right.$in $\left.\mathrm{cm}^{-1}\right)$.

\begin{tabular}{lccccccc}
\hline \hline \multicolumn{3}{c}{ Experiment } & \multicolumn{5}{c}{ Theory } \\
& $\omega$ & $g$ & $\omega$ & $g$ & $g(L S)$ & $q_{-}$ & $q_{+}$ \\
${ }^{6} D_{9 / 2}^{o}$ & 38459 & 1.542 & 38352 & & 1.556 & 1359 & 1363 \\
${ }^{6} D_{7 / 2}^{o}$ & 38660 & 1.584 & 38554 & 1.586 & 1.587 & 1522 & 1510 \\
${ }^{6} F_{11 / 2}^{o}$ & 41968 & & 41864 & & 1.455 & 1496 & 1508 \\
${ }^{6} F_{9 / 2}^{o}$ & 42115 & 1.43 & 42012 & & 1.434 & 1615 & 1631 \\
${ }^{6} F_{7 / 2}^{o}$ & 42237 & 1.399 & 42141 & 1.396 & 1.397 & 1738 & 1737 \\
${ }^{6} P_{7 / 2}^{o}$ & 42658 & 1.702 & 42715 & 1.709 & 1.714 & 1241 & 1261 \\
${ }^{4} D_{7 / 2}^{o}$ & 44447 & 1.40 & 44600 & 1.345 & 1.429 & 1791 & 1837 \\
${ }^{4} F_{7 / 2}^{o}$ & 44754 & 1.29 & 44386 & 1.327 & 1.238 & 1608 & 1601 \\
${ }^{8} P_{7 / 2}^{o}$ & 54490 & & 54914 & 1.936 & 1.937 & -2084 & -2086 \\
${ }^{4} G_{7 / 2}^{o}$ & 60957 & 0.969 & 63624 & 0.978 & 0.984 & 1640 & 1640 \\
${ }^{4} H_{7 / 2}^{o}$ & 61157 & 0.720 & 63498 & 0.703 & 0.667 & 1296 & 1247 \\
${ }^{4} D_{7 / 2}^{o}$ & 61726 & 1.411 & 66145 & 1.398 & 1.429 & 1194 & 1240 \\
${ }^{4} F_{7 / 2}^{o}$ & 62066 & 1.198 & 65528 & 1.252 & 1.238 & 1071 & 1052 \\
${ }^{6} P_{7 / 2}^{o}$ & 62172 & 1.68 & 65750 & 1.713 & 1.714 & -1524 & -1514 \\
${ }^{2} G_{7 / 2}^{o}$ & 62323 & & 64798 & 0.882 & 0.889 & 1622 & 1605 \\
\hline \hline
\end{tabular}

affect the slopes $q$.

Five levels from this band belong to configuration $3 d^{6} 4 p$ and have close slopes with the average $\bar{q}=$ $1360 \mathrm{~cm}^{-1}$. Only the level ${ }^{4} F_{7 / 2}^{o}$ has the slope, which is $300 \mathrm{~cm}^{-1}$ smaller, than the average. The remaining level ${ }^{6} P_{7 / 2}^{o}$ belongs to configuration $3 d^{5} 4 s 4 p$ and has the slope of the opposite sign $q_{1}=-1519 \mathrm{~cm}^{-1}$. Its absolute value is $500 \mathrm{~cm}^{-1}$ smaller, than for the level ${ }^{8} P_{7 / 2}^{o}$ of the same configuration $3 d^{5} 4 s 4 p$. That suggests that the levels ${ }^{4} F_{7 / 2}^{o}$ and ${ }^{6} P_{7 / 2}^{o}$ strongly interact with each other. This is also in agreement with the fact, that these levels are the closest neighbors both experimentally and theoretically and that they cross somewhere between $x_{-}$and $x$. There is also strong interaction between the levels ${ }^{2} G_{7 / 2}^{o},{ }^{4} F_{7 / 2}^{o}$, and ${ }^{4} D_{7 / 2}^{o}$. That can be seen if one calculates the scalar products (overlaps) between corresponding wave functions for different values of $x$, such as: $\left\langle i\left(x_{-}\right) \mid k\left(x_{+}\right)\right\rangle$. For weekly interacting levels $\left\langle i\left(x_{-}\right) \mid k\left(x_{0}\right)\right\rangle \approx\left\langle i\left(x_{-}\right) \mid k\left(x_{+}\right)\right\rangle \approx \delta_{i, k}$, so large nondiagonal matrix elements signal, that corresponding levels interact.

Interaction of levels ${ }^{2} G_{7 / 2}^{o},{ }^{4} F_{7 / 2}^{o}$, and ${ }^{4} D_{7 / 2}^{o}$ does not affect the slopes $q$ as strongly, as the interaction of ${ }^{4} F_{7 / 2}^{o}$ and ${ }^{6} P_{7 / 2}^{o}$, so we can account for the former in a less accurate way, but it is important to include the latter as accurately as possible.

The level ${ }^{6} P_{7 / 2}^{o}$ interacts with some linear combination of levels ${ }^{2} G_{7 / 2}^{o},{ }^{4} F_{7 / 2}^{o}$, and ${ }^{4} D_{7 / 2}^{o}$. The slopes and $g$-factors of the latter are relatively close to each other, so we can simply take the average for all three:

$$
\bar{g}=1.185 ; \quad \bar{q}=1297 .
$$

Now we can use experimental $g$-factor of the state ${ }^{6} P_{7 / 2}^{o}$

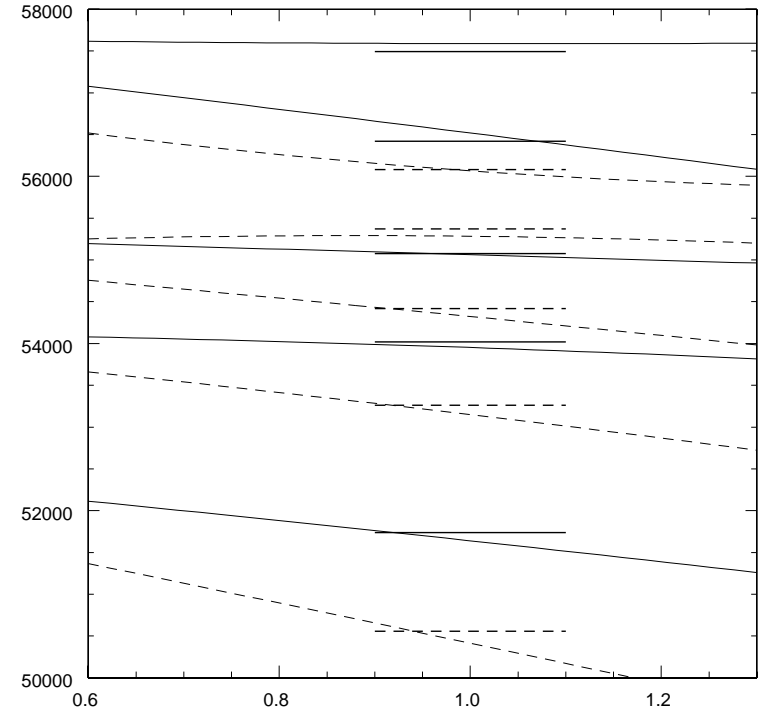

FIG. 2: Dependence of the odd levels of Ni II on $\left(\alpha / \alpha_{0}\right)^{2}=$ $x+1$. Solid lines correspond to $J=5 / 2$ and dashed lines to $J=7 / 2$. The experimental positions of the lines are shown as short horizontal lines and are all shifted by $1000 \mathrm{~cm}^{-1}$. The order of levels from bottom up: ${ }^{4} D_{7 / 2,5 / 2}^{o},{ }^{4} G_{7 / 2,5 / 2}^{o},{ }^{4} F_{7 / 2,5 / 2}^{o}$, ${ }^{2} G_{7 / 2}^{o},{ }^{2} F_{5 / 2}^{o},{ }^{2} D_{5 / 2}^{o}$, and ${ }^{2} F_{5 / 2}^{o}$.

and Eq. (11) to determine the mixing:

$$
\begin{aligned}
& C^{2}=\frac{1.68-\bar{g}}{1.713-\bar{g}}=0.937, \\
& q\left({ }^{6} P_{7 / 2}^{o}\right)=-1342 .
\end{aligned}
$$

Eq. (15) corresponds to the correction $\delta q=+177$. Therefore, for the closest level ${ }^{4} F_{7 / 2}^{o}$ this model gives an estimate:

$$
q\left({ }^{4} F_{7 / 2}^{o}\right)=\bar{q}-\delta q=1120 .
$$

Eqs. (15) and (16) show that correction for the mixing is not very large. That corresponds to the fact that experimental $g$-factor of the level ${ }^{6} P_{7 / 2}^{o}$ is significantly larger than any $g$-factors of the levels of the configuration $3 d^{6} 4 p$. Thus, the interaction for this level is relatively small. On the contrary, the levels of the configuration $3 d^{6} 4 p$ strongly interact with each other, but corresponding changes of the slopes are also relatively small (since the $q$ values for these strongly interacting levels are approximately the same).

We estimate the accuracy of our calculations for the lower band of Fe II to be about $150 \mathrm{~cm}^{-1}$, and approximately $300 \mathrm{~cm}^{-1}$ for the values (15) and (16).

\section{E. Ni II}

Ni II has the ground state configuration $3 d^{9}$. The spectrum is somewhat simpler, than for Fe II. There are also 
TABLE VI: Transition frequencies $\omega$ from the ground state ${ }^{2} D_{5 / 2}, g$-factors, and parameters $q_{ \pm}$for Ni II $\left(\right.$in $\left.\mathrm{cm}^{-1}\right)$.

\begin{tabular}{lcccccrrr}
\hline \hline & \multicolumn{6}{c}{ Experiment } & \multicolumn{5}{c}{ Theory } \\
& $\omega$ & $g$ & $\omega$ & $g$ & $g(L S)$ & $q_{-}$ & $q_{+}$ \\
${ }^{2} D_{3 / 2}$ & 1507 & & 1579 & & 0.800 & 1559 & 1552 \\
${ }^{4} D_{7 / 2}^{o}$ & 51558 & 1.420 & 50415 & 1.423 & 1.429 & -2405 & -2425 \\
${ }^{4} D_{5 / 2}^{o}$ & 52739 & 1.356 & 51640 & 1.360 & 1.371 & -1217 & -1245 \\
${ }^{4} G_{7 / 2}^{o}$ & 54263 & 1.02 & 53150 & 1.016 & 0.984 & -1334 & -1387 \\
${ }^{4} G_{5 / 2}^{o}$ & 55019 & 0.616 & 53953 & 0.617 & 0.571 & -370 & -418 \\
${ }^{4} F_{7 / 2}^{o}$ & 55418 & 1.184 & 54323 & 1.183 & 1.238 & -1104 & -1124 \\
${ }^{4} F_{5 / 2}^{o}$ & 56075 & 0.985 & 55063 & 0.986 & 1.029 & -332 & -334 \\
${ }^{2} G_{7 / 2}^{o}$ & 56372 & 0.940 & 55284 & 0.933 & 0.889 & -60 & -188 \\
${ }^{2} F_{7 / 2}^{o}$ & 57080 & 1.154 & 56067 & 1.128 & 1.143 & -911 & -713 \\
${ }^{2} D_{5 / 2}^{o}$ & 57420 & 1.116 & 56520 & 1.108 & 1.200 & -1419 & -1438 \\
${ }^{2} F_{5 / 2}^{o}$ & 58493 & 0.946 & 57589 & 0.959 & 0.857 & -35 & -5 \\
\hline \hline
\end{tabular}

pseudo-crossings here, but they either lie far from $x=0$, or are rather wide. That makes their treatment slightly easier. Nevertheless, our results significantly differ from previous calculations [28].

CI calculations were done for the Coulomb potential and included SD and partly triple excitations on the basis set $[5 \mathrm{spdf}]$. We calculated 5 lower odd levels with $J=5 / 2$ and 5 with $J=7 / 2$ for $x_{-}, x_{0}$, and $x_{+}$, and used parabolic extrapolation for the interval $-0.4 \leq x \leq+0.3$ (see Fig. 2). It is seen that the theory accurately reproduce relative positions of all levels. An overall agreement between the theory and the experiment becomes close to perfect if all experimental levels are shifted by $1000 \mathrm{~cm}^{-1}$ down, as it is done in Fig. 2 Note, that this shift constitutes only $2 \%$ of the average transition frequency.

Calculated $g$-factors are generally in agreement with the experiment [35] and noticeably different from the pure $L S$-values (see Table VI). However, for the level ${ }^{2} F_{7 / 2}^{o}$ theoretical $g$-factor is smaller than the $L S$ value, while experimental one is larger. There are no nearby levels who may mix to this one and move $g$-factor closer to experiment. On the other hand, the difference with experiment is only $2 \%$ and may be within experimental accuracy.

Fig. 2 shows that the levels ${ }^{2} G_{7 / 2}^{o}$ and ${ }^{2} F_{7 / 2}^{o}$ cross at $x \approx 0.3$ and they already strongly interact at $x=0$. Theoretical splitting for these levels is $10 \%$ larger than experimental one. Thus, they are in fact even closer to the crossing point than is predicted by the theory. The experimental splitting is equal to the theoretical one for larger value of $\alpha$ corresponding to $x \approx 0.15$. At $x=0.15$ the slopes of these levels are -265 and -590 , and for $x=$ 0 they are -124 and -812 correspondingly. Note, that the sum of the slopes at $x=0.15$ differs by $80 \mathrm{~cm}^{-1}$ from the sum at $x=0$. According to Eq. (7) for a two-level system the sum is constant. This means that these two levels repel from the lower lying level ${ }^{4} F_{7 / 2}^{o}$. Taking this analysis into account we suggest an average between $x=$ 0 and $x=0.15$ as our final value: $q\left({ }^{2} F_{7 / 2}^{o}\right)=-700(250)$.

\section{Conclusions}

In this paper we present new refined calculations of the parameters $q$, which determine $\alpha$-dependence of the transition frequencies for a number of ions used in the astrophysical search for $\alpha$-variation. These ions appear to be very different from the theoretical point of view. Because of that we had to use different methods and different levels of approximation for them. The final accuracy of our results differs not only for different ions, but also for different transitions.

The simplest system is Zn II, which has one valence electron. On the other hand, this is the heaviest ion and it has the largest core, which includes $3 d^{10}$-shell. That gave us the opportunity to study corrections to $q$ from the core-valence correlations and from Breit interaction. We found the former to be about $17 \%$ and the latter to be less than $1 \%$. For lighter ions Breit interaction should be even smaller and can be safely neglected. Other ions also have much smaller and more rigid cores, so one might expect that core-valence correlations are few times weaker there in comparison to $\mathrm{Zn}$. That allows us to neglect corevalence correlations for all other ions discussed in this paper.

Si II has the smallest core $1 s^{2} \ldots 2 p^{6}$ and three valence electrons. For neutral $\mathrm{Mg}$, which has the same core, the core-valence corrections to the $3 s \rightarrow 3 p$ transition frequencies were found to be about $4 \%$ [33, 36]. CI calculation for Si II is relatively simple and the errors associated with incompleteness of CI space are small. Thus, our estimate of the accuracy for $\mathrm{Si}$ on $6 \%$ level seems to be rather conservative.

$\mathrm{Cr}, \mathrm{Fe}$, and $\mathrm{Ni}$ have the core $1 s^{2} \ldots 3 p^{6}$ and the core excitation energy varies from 2 a.u. for Cr II to 2.6 a.u. for Ni II. In comparison, the core excitation energy for $\mathrm{Zn}$ II is 0.9 a.u. Therefore, we estimate the core-valence correlation corrections for these ions to be at least two times smaller, than for Zn II.

Additional error here is associated with incompleteness of the CI space. These ions have from 5 to 9 valence electrons and CI space can not be saturated. To estimate corresponding uncertainty we performed several calculations for each ion using different basis sets and two different computer packages described in Sec. III The basic Dirac-Hartree-Fock orbitals were calculated for different configurations (for example, for the ground state configuration and for excited state configuration, etc.).

Supplementary information on the accuracy of our calculations can be obtained from comparison of calculated spectra and $g$-factors with experimental values. The later appear to be very important as they give information about electron coupling, which depends on relativistic corrections and on interaction between $L S$-multiplets. Our results for Cr II appear to be very close for different calculations and are in good agreement with the experiment both in terms of the gross level structure and spin-orbit splittings (see Table IV], so we estimate our final error here to be about $10-12 \%$. 
The largest theoretical uncertainties appear for Fe II and Ni II where the number of valence electrons is largest and the interaction of levels is strongest. Here we had to include semi-empirical fits to improve the agreement between the theory and the experiment. We took into account the size of these semi-empirical corrections in estimates of the accuracy of the calculated values of $q$.

The final results are presented in Table【 Note again, that they are based on several independent calculations performed using two different computer codes. Some of the intermediate results are given in Table [II- Table VI

\section{Acknowledgments}

This work is supported by Australian Research Council. One of us (MK) thanks UNSW for hospitality and acknowledges support from the Gordon Godfrey Fund.
[1] J. K. Webb, V. V. Flambaum, C. W. Churchill, M. J. Drinkwater, and J. D. Barrow, Physical Review Letters 82, 884 (1999).

[2] J. K. Webb, M. T. Murphy, V. V. Flambaum, V. A. Dzuba, J. D. Barrow, C. W. Churchill, J. X. Prochaska, and A. M. Wolfe, Phys. Rev. Lett. 87, 091301 (2001).

[3] M. T. Murphy, J. K. Webb, V. V. Flambaum, V. A. Dzuba, C. W. Churchill, J. X. Prochaska, J. D. Barrow, and A. M. Wolfe, Mon. Not. R. Astron. Soc. 327, 1208 (2001).

[4] M. T. Murphy, J. K. Webb, V. V. Flambaum, C. W. Churchill, and J. X. Prochaska, Mon. Not. R. Astron. Soc. 327, 1237 (2001).

[5] M. T. Murphy, J. K. Webb, V. V. Flambaum, M. J. Drinkwater, F. Combes, and T. Wiklind, Mon. Not. R. Astron. Soc. 327, 1244 (2001).

[6] M. T. Murphy, J. K. Webb, V. V. Flambaum, C. W. Churchill, and J. X. Prochaska, Mon. Not. R. Astron. Soc. 327, 1223 (2001).

[7] F. Combes and T. Wiklind, Astron. Soc. Pacific. Astronomical Society of the Pacific Conference Series 156, 210 (1999).

[8] A. V. Ivanchik, A. Y. Potekhin, and D. A. Varshalovich, Astronomy \& Astrophysics 343, 439 (1999).

[9] D. A. Varshalovich, A. V. Ivanchik, and A. Y. Potekhin, Zhurnal Tekhnicheskoi Fiziki 69, 1 (1999).

[10] J. D. Prestage, R. L. Tjoelker, and L. Maleki, AIP. American Institute of Physics Conference Proceedings (457), 357 (1999).

[11] Y. Sortais, S. Bize, C. Nicolas, M. Santos, C. Mandache, G. Santarelli, C. Salomon, and A. Clairon, Proceedings of the 1999 Joint Meeting of the European Frequency and Time Forum and the IEEE International Frequency Control Symposium. IEEE. 1, 34 (1999).

[12] Y. Sortais, S. Bize, C. Nicolas, G. Santarelli, G. S. C. Salomon, and A. Clairon, IEEE Transactions on Ultrasonics Ferroelectrics \& Frequency Control 47, 1093 (2000).

[13] C. Braxmaier, O. Pradl, H. Muller, A. Peters, J. Mlynek, V. Loriette, and S. Schiller, Physical Review D 64, 042001 (2001).

[14] M. Kaplinghat, R. J. Scherrer, and M. S. Turner, Physical Review D 60, 023516 (1999).

[15] S. Hannestad, Physical Review D 60, 023515 (1999).

[16] J. Kujat and R. J. Scherrer, Physical Review D 62,
023510 (2000).

[17] C. Alvarez and R. B. Mann, Physical Review D 55, 1732 (1997).

[18] L. Bergstrom, S. Iguri, and H. Rubinstein, Physical Review D 60, 045005 (1999).

[19] R. W. Kuhne, Modern Physics Letters A 14, 1917 (1999).

[20] N. Chamoun, S. J. Landau, and H. Vucetich, Physics Letters B 504, 1 (2001).

[21] D. Kabat and A. Rajaraman, Physics Letters B 516, 383 (2001).

[22] Y. Fujii, Phys. Rev. D 62, 064004 (2000).

[23] H. Sandvik, J. D. Barrow, and J. Magueijo, $A$ simple varying-alpha cosmology (2001), e-print: astro-ph/0107512

[24] I. I. Sobelman, Atomic spectra and radiative transitions (Springer-Verlag, Berlin, 1979).

[25] V. A. Dzuba, V. V. Flambaum, and J. K. Webb, Physical Review Letters 82, 888 (1999).

[26] V. A. Dzuba, V. V. Flambaum, and J. K. Webb, Physical Review A 59, 230 (1999).

[27] V. A. Dzuba and V. V. Flambaum, Physical Review A 61, 034502 (2000).

[28] V. A. Dzuba, V. V. Flambaum, M. T. Murphy, and J. K. Webb, Phys. Rev. A 63, 042509 (2001).

[29] E. Lindroth, A.-M. Mårtensson-Pendrill, A. Ynnerman, and P. Öster, J. Phys. B 22, 2447 (1989).

[30] V. A. Dzuba, V. V. Flambaum, and M. G. Kozlov, Phys. Rev. A 54, 3948 (1996).

[31] V. A. Dzuba and W. R. Johnson, Phys. Rev. A 57, 2459 (1998).

[32] M. G. Kozlov and S. G. Porsev, Opt. Spectrosc. 87, 352 (1999).

[33] S. G. Porsev, M. G. Kozlov, Y. G. Rakhlina, and A. Derevianko, Phys. Rev. A 64, 012508 (2001).

[34] P. Bogdanovich and G. Žukauskas, Sov. Phys. Collection 23(5), 18 (1983).

[35] C. E. Moore, Atomic Energy Levels, vol. 1-3 (Natl. Bur. Stand. (US), Circ. No. 467, Washington, 1958).

[36] S. G. Porsev, M. G. Kozlov, and Y. G. Rahlina, JETP Lett. 72, 595 (2000).

[37] In fact, the frequency $\omega\left(n p_{1 / 2} \rightarrow n p_{3 / 2}\right)$ is not measured directly, but is found as a difference: $\omega\left(n^{\prime} s_{1 / 2} \rightarrow n p_{3 / 2}\right)-$ $\omega\left(n^{\prime} s_{1 / 2} \rightarrow n p_{1 / 2}\right)$. 